\title{
Implementation and Validation of Schapery-Rand Anisotropic Viscoelasticity Model for Super-Pressure Balloons
}

\author{
T. Gerngross* and S. Pellegrino ${ }^{\dagger}$ \\ University of Cambridge, Cambridge, CB2 1PZ, UK
}

\begin{abstract}
The thin film used for the NASA Ultra Long Duration Balloons (ULDB) shows considerable time-dependent behaviour. Furthermore, experiments on scaled ULDB balloons have revealed that wrinkles are present over a wide range of pressures. A numerical model has been developed describing the nonlinear anisotropic viscoelastic material behaviour by means of a Schapery-type model and this model has been extended to model wrinkling by means of a user-defined subroutine in the finite-element package ABAQUS. After a description of the viscoelastic modelling approach, a lobe of a 48 gore ULDB flat facet balloon is modelled and compared to experimental results. Additionally two test cases of anisotropic wrinkling are presented, one involving a flat membrane and one a cylindrical balloon structure.
\end{abstract}

\section{Introduction}

Large super-pressure stratospheric balloons currently under development in the NASA Ultra Long Duration Balloon (ULDB) Program make use of thin polymeric films to form a sealed envelope that is constrained by a series of meridional tendons. In the balloon the polymeric film is subject to a biaxial state of stress whose details depend on the cutting pattern, stiffness of the film vs. stiffness of the tendons, etc. Viscoelastic effects, which are usually significant in the film, play a significant role in the stress distribution and shape of these balloons.

So far pseudo-elastic material properties have been generally assumed for the design of the balloon structure. However, following a number of anomalies during flight tests of NASA Ultra-Long-DurationBalloons (ULDB), ${ }^{1}$ it has been realized that the behaviour of super-pressure balloons is more complex than assumed at first. As the complexity of these balloons is better grasped, ${ }^{11,20}$ detailed experimental validation of the analysis models is being initiated, and this in turn requires that details of the time-dependent material behavior be also included in the models. A finite element model of Schapery's nonlinear viscoelastic material model has been developed ${ }^{3,4}$ and verified by means of cylindrical balloon structures that provide uniform states of stress. Further investigation of more complex states of stress is needed in order to enable realistic models of ULDBs. In a first attempt this paper presents predictions for creep strains on a ULDB 48 gore flat facet balloon that are compared to experimental results obtained in reference 22 .

Experiments on small scale ground models with 48 gores, nominal 4 m diameter, and a constant lobe radius design showed partially wrinkled regions over wide ranges of pressures. With increasing pressures and/or after some time under pressurisation these wrinkles eventually disappeared. A ULDB 48 gore flat facet balloon has shown the presence of wrinkles up to a differential pressure of $500 \mathrm{~Pa}^{2}$

While a wrinkled surface may cause difficulties during experimental measurements the challenge for finite element models is even higher. The formation of wrinkles is associated with (small) compressive stresses; these stresses cannot be carried by membrane elements and consequently numerical instabilities occur. Since in general most balloon structures develop wrinkles in some regions, at least during pressurisation it is important to take wrinkling into consideration.

This paper is part of an ongoing effort to develop more realistic models for super-pressure balloons, validated with reference to representative physical models. Here we present an approach to model both

\footnotetext{
*Research student, Department of Engineering, Trumpington Street.

${ }^{\dagger}$ Professor of Structural Engineering, Department of Engineering, Trumpington Street. Associate Fellow AIAA.
} 


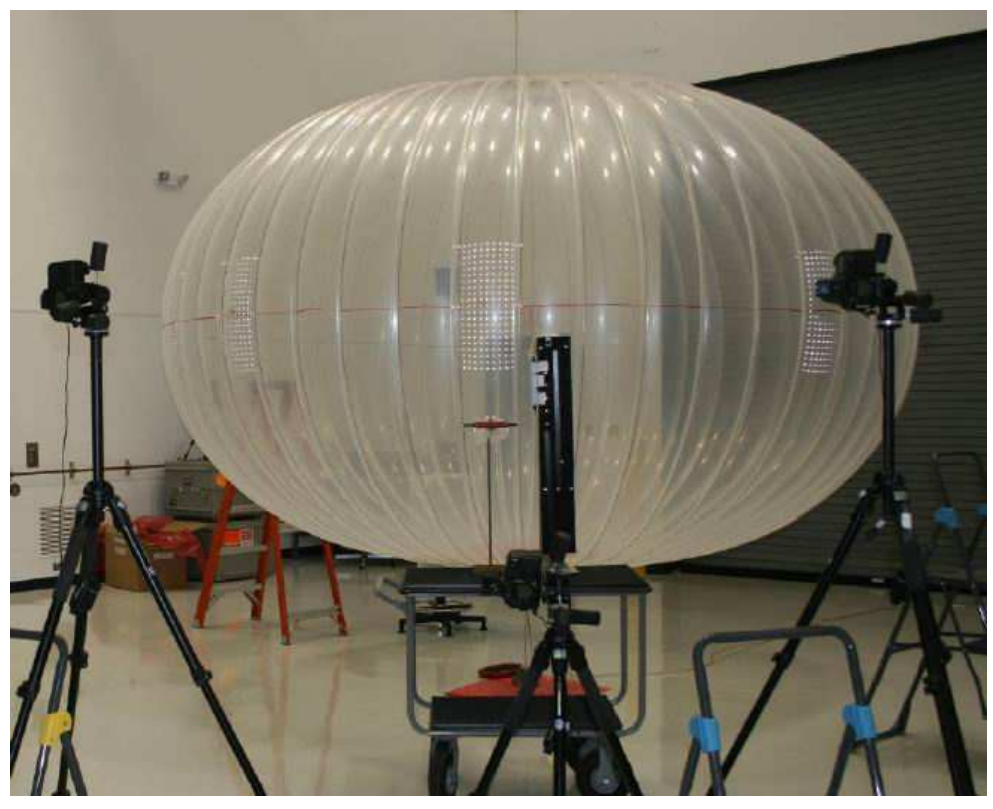

Figure 1. ULDB 48 gore flat facet balloon, from Young et al. ${ }^{22}$

nonlinear viscoelasticity and anisotropic wrinkling implemented through a user-defined material subroutine in the ABAQUS finite-element package. Based on this approach, we have predicted the creep strains in a flat facet balloon and compared to experimental results. Additionally an approach to model anisotropic wrinkling is presented. It is then attempted to combine both approaches into one model, which is tested on a cylindrical balloon structure.

\section{Background}

\section{II.A. Material}

A linear low density polyethylene (LLDPE) film, called StratoFilm 372, has been used for many years for NASA balloons. This film was produced as a monolayer extrusion with a blow-up-ratio (BUR) of three, and a thickness of $0.02 \mathrm{~mm}$ was achieved. This material has been fully characterised, resulting in a detailed time-dependent material description. ${ }^{6,12,13}$

For the ULDB a new material, called StratoFilm 430, has been introduced. This consists of a three layer co-extrusion using the same LLDPE resin as for SF372. A different die and a reduced BUR have resulted in a film with a thickness of $0.038 \mathrm{~mm}$. It has been suggested that these changes affect only the transverse direction properties of the film and so the properties in the machine direction remain unchanged. ${ }^{12}$ After some initial creep tests we decided to use Rand's master curve and nonlinearity functions for SF372.

\section{II.B. Viscoelastic Model}

A general introduction to the field of nonlinear viscoelasticity is provided in standard textbooks. ${ }^{8,21}$ In reference 3 we have presented an attempt to model the time-dependent material behavior of LLDPE using the creep/relaxation models available in ABAQUS. Additionally the Schapery ${ }^{16}$ nonlinear viscoelastic constitutive material model has been implemented as a user defined material (UMAT) for the use in ABAQUS and verified by means of cylindrical balloon structures. ${ }^{3,4}$ This alternative approach is quite accurate and will be being used in the following sections.

Schapery's material mode ${ }^{16}$ is based on the thermodynamics of irreversible processes, where the transient material behavior is defined by a master creep function. Nonlinearities can be considered by including factors that are functions of stress and temperature. Further, horizontal shift factors enable coverage of wide temperature/stress ranges. Schapery also gave a general multiaxial formulation with the nonlinear function being an arbitrary function of stress. Since the Poisson's ratio has only a weak time-dependence a single time-dependent function is sufficient to characterize all elements of the linear viscoelastic creep compliance 
matrix. ${ }^{17}$

Rand and co-workers ${ }^{12,14}$ further simplified this relationship by assuming that the time-dependence in any material direction is linearly related to that observed in the machine direction:

$$
\varepsilon_{i}^{t}=g_{0}^{t} S_{i j}^{0} D_{0} \sigma_{j}^{t}+g_{1}^{t} \int_{0}^{t} S_{i j} \Delta D^{\left(\psi^{t}-\psi^{\tau}\right)} \frac{d\left(g_{2}^{\tau} \sigma_{j}^{\tau}\right)}{d \tau} d \tau
$$

where the reduced time is

$$
\psi^{t}=\int_{0}^{t} \frac{d \tau}{a_{\sigma}(T, \sigma) a_{T}(T)}
$$

The first term in Equation (1) represents the elastic response of the material, provided by the instantaneous elastic compliance $D_{0}$, while the second term describes the transient response, defined by the transient compliance function, $\Delta D$. The other parameters are nonlinearity functions and the horizontal shift factors for the master-curve.

$S_{i j}^{0}$ and $S_{i j}$ are the coefficient matrices enabling the multiaxial formulation. Since the material response in any direction is based on the properties in the machine direction, one assumes $S_{11}^{0}=1$ and $S_{11}=1$. Anisotropic behaviour is accounted for by adjusting the remaining coefficients.

\section{Viscoelastic Subroutine}

To use Schapery's single-integral constitutive model in a numerical algorithm, Equation (1) needs to be rewritten in incremental form. A numerical integration method was presented by Haj-Ali and Muliana ${ }^{5}$ for a three-dimensional, isotropic material. Based on the integration method proposed in reference 5, an algorithm has been developed for anisotropic material behavior that implements the biaxial approach of Rand and co-workers. ${ }^{12,14}$ This algorithm was implemented in ABAQUS, but would be equally suitable for any displacement based finite element software, where strain components are used as the independent state variables. $^{3,4}$

The incremental method requires the transient strain function $\Delta D$ to be expressed in terms of a sum of exponentials, called a Prony series, and the strain/stress history needs to be stored at the end of each increment for each strain/stress component and each Prony term. A schematic overview of the iterative algorithm is depicted in Figure 2 while the full set of equations has been presented in reference 4 .

The ABAQUS interface for a user-defined material (UMAT) passes the current time increment $\Delta t$ and

the corresponding strain increment $\Delta \varepsilon$, determined using the Jacobian matrix, $\partial \sigma_{j}^{t} / \partial \varepsilon_{i}^{t}$, at the end of the previous time increment. In turn, it requires at the end of the current time increment an update of the stresses $\sigma_{j}^{t}$ and the updated Jacobian matrix.

Every time UMAT is called, it starts with an estimation of a trial stress increment $\Delta \sigma^{t, t r i a l}$ based on the nonlinearity parameters at the end of the previous time increment. With this initial guess an iterative loop is entered, where the integration in Equation (1) yields $\Delta \varepsilon^{t, t r i a l}$, which is compared with $\Delta \varepsilon^{t, A B A Q U S}$. If required, the stresses and the nonlinearity parameters are corrected and the loop is repeated. Alternatively, if the strain error residual is below a specified tolerance ( the Jacobian matrix and the stresses.

The subroutine has been verified for cylindrical balloon structures, ${ }^{4}$ without any wrinkles. An approach that allows for the presence of wrinkles in thin anisotropic membranes is presented in the following section.

\section{Wrinkling of Anisotropic Membranes}

Thin membranes cannot carry compressive stresses and consequently wrinkles or slack regions form. Most balloon structures develop wrinkles, at least during pressurisation and especially near any end fittings and tendons. Experimental observations showed that wrinkles appear even in a flat facet balloon structure. ${ }^{2}$ In order to allow for experimental validation of numerical models it is important to consider the effects of wrinkling in thin anisotropic material. In the following we present a first attempt to predict the correct stresses and displacements in a partially wrinkled anisotropic membrane surface. Our aim is not to model the exact shape of the wrinkles but rather the average surface as shown in Figure 3(a). Also the following is limited to flat membranes with in-plane loading. For a wrinkled state we make the following assumptions: the bending stresses in the membrane are negligible, the stress in the wrinkle direction is zero, and there is a uniaxial stress in perpendicular direction. 


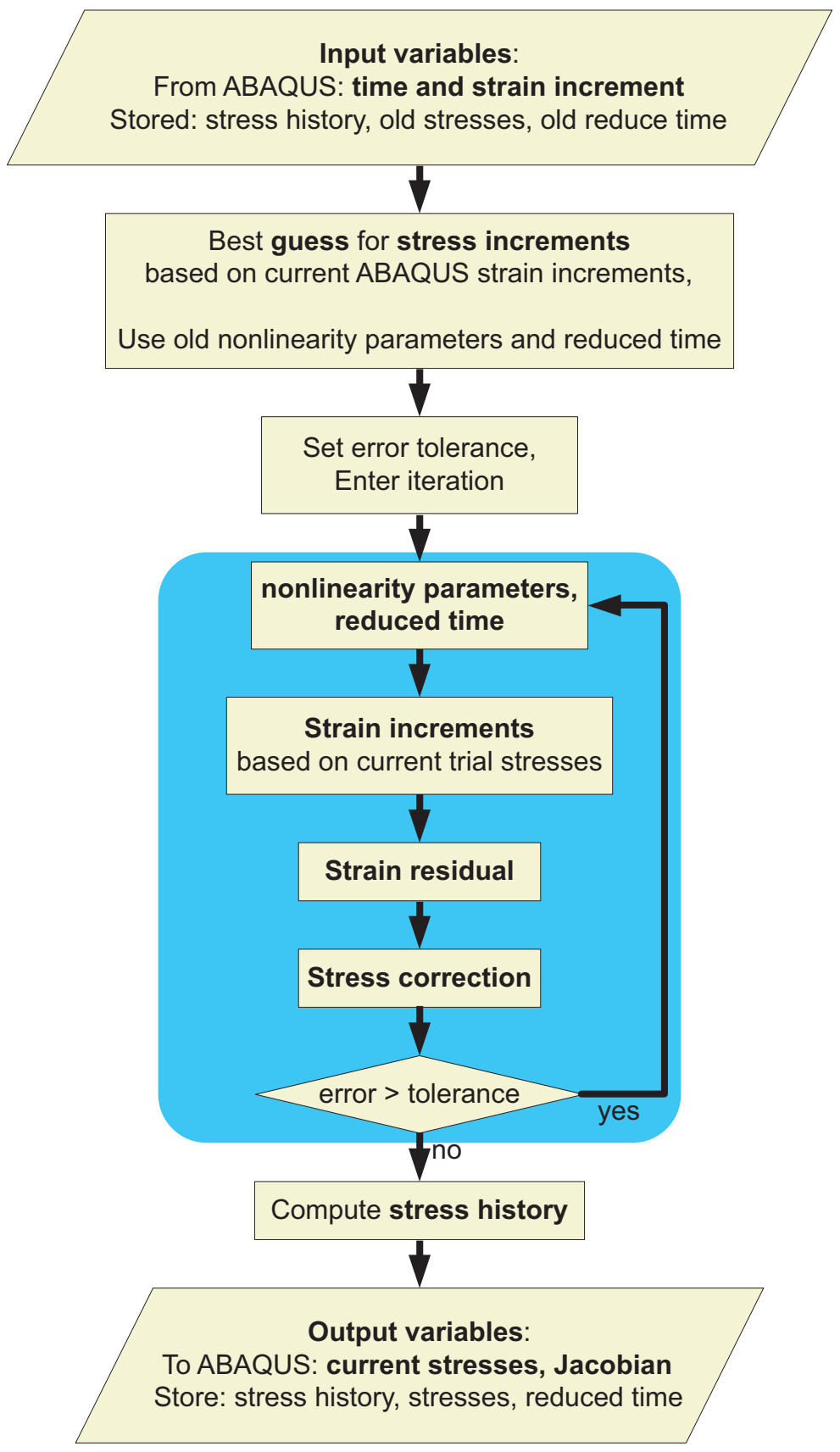

Figure 2. Schematic overview of the user-defined subroutine. 


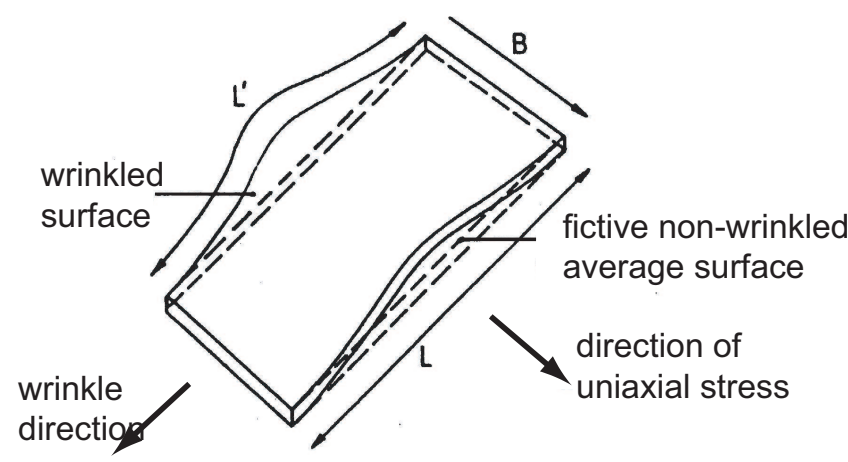

(a) deformed configuration

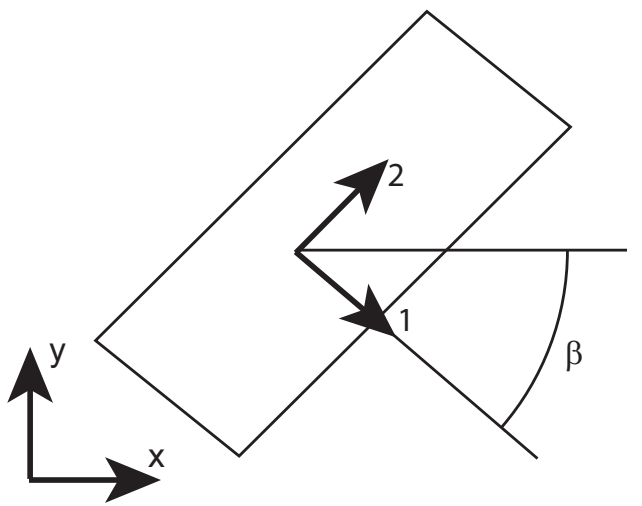

(b) undeformed configuration

Figure 3. Average wrinkling surface, from Roddeman et al. ${ }^{15}$

A general approach to consider wrinkles in isotropic materials was proposed by Stein and Hedgepeth ${ }^{18}$ and further developed in references 9,10. It was observed that when wrinkles develop within a membrane the associated overall contraction in the direction of the wrinkles exceeds that predicted by the Poisson's ratio effect. Hence, this approach requires an appropriate increase of the local effective value of the Poisson's ratio in wrinkled regions. This effective value of Poisson's ratio is determined by imposing a locally uniaxial stress state in the direction of the wrinkles. However, in order to apply the Stein and Hedgepeth approach the wrinkling orientation must be known, and while for isotropic materials the direction of the wrinkles is determined by the principal stresses with any shear components being zero, this is generally not the case for anisotropic materials. Hence, the approach described in the following subsections consists of the following steps:

1. check the element state: taut, slack or wrinkled;

2. if wrinkled determine the wrinkling orientation;

3. find an effective elasticity matrix that leads to a compression-free state of stress.

\section{IV.A. Wrinkling Criterion}

Kang and $\operatorname{Im}^{7}$ used a combined stress-strain criterion based on principal stresses and strain to determine if an element is wrinkled or not. Three different states were considered:

$$
\begin{array}{lc}
\text { principal stress } \sigma_{22}>0 & \text { taut } \\
\text { principal strain } \varepsilon_{11} \leq 0 & \text { slack } \\
\text { otherwise }\left(\sigma_{22} \leq 0 \text { and } \varepsilon_{11}>0\right) & \text { wrinkled }
\end{array}
$$

If the element is found to be taut no further action is required and in case of a slack state all stresses are set to zero and an empty elasticity matrix is required. For a wrinkled element the following sections describe the procedure.

\section{IV.B. Wrinkling Orientation}

Kang and $\operatorname{Im}^{7}$ have presented a scheme where the orientation and the magnitude of the uniaxial tension is obtained from an invariant relationship between the normal strain component in the direction of the local uniaxial tension and the shear strain component. Presuming a uniaxial state of stress, they derived the following two relationships for the strain components $\varepsilon_{22 u}$ and $\varepsilon_{12 u}$, where the subscript $u$ denotes directions parallel and perpendicular to the wrinkle, defined on the actual wrinkled surface, as depicted in Figure 3(a):

$$
\varepsilon_{22 u}=\frac{C_{12} C_{33}-C_{23} C_{31}}{C_{23} C_{32}-C_{22} C_{33}} \cdot \varepsilon_{11 u}
$$




$$
2 \varepsilon_{12 u}=\frac{C_{22} C_{31}-C_{21} C_{32}}{C_{23} C_{32}-C_{22} C_{33}} \cdot \varepsilon_{11 u}
$$

where $C_{i j}$ are the components of the stress-strain relations after a rotation of the coordinate system by an angle $\beta$, see Figure 3(b). They found that Equation (4) is an invariant with respect to the magnitude of wrinkling, valid for both wrinkled states and (unwrinkled) uniaxial tension, while Equation (3) holds only for uniaxial tension. Based on these two equations they propose a scheme to determine the wrinkling orientation, i.e. the direction of the uniaxial tension.

Starting from an initial guess for the angle $\beta$ that defines the wrinkle orientation, the coordinate system is rotated into the local 1,2-system and the following procedure is applied:

1. in the local 1,2 -system check that $\varepsilon_{11}>0$ for the assumed value of $\beta$

2. set $\varepsilon_{11 u}=\varepsilon_{11}$

3. calculate $\varepsilon_{12 u}$ and $\varepsilon_{22 u}$ from Equations (3),(4)

4. $\beta$ defines the wrinkling orientation if $\varepsilon_{12}=\varepsilon_{12 u}$ and $\varepsilon_{22 u} \geq \check{\varepsilon}_{22}$

where the components $\varepsilon_{i j}$ are the strains from a rotation of the coordinate system. The key in the above procedure is to find an angle $\beta$ that solves the equation $\varepsilon_{12 u}-\varepsilon_{12}=0$.

\section{IV.C. Effective Elasticity Matrix}

While in a taut element stresses and strains are related by the standard relationship for plane stress elasticity, in a wrinkled region these equations do not apply. Once the correct wrinkling orientation has been found a new effective elasticity matrix $D_{\text {eff }}$ can be determined that relates a given state of strain to a state of uniaxial stress. The derivation of $D_{\text {eff }}$ follows Miller et al. ${ }^{10}$

Assuming negligible bending stress in the membrane and imposing uniaxial stress yields for stresses in wrinkling orientation

$$
\begin{aligned}
& \sigma_{11}=C_{11} \varepsilon_{11}+C_{12} \varepsilon_{22}+C_{13} \gamma_{12} \\
& \sigma_{22}=0 \\
& \sigma_{12}=0
\end{aligned}
$$

For uniaxial stress in the 1-direction $\varepsilon_{22}$ and $\gamma_{12}$ in Equation (5) can be eliminated using the strain-stress relationship and $\sigma_{11}$ can be rewritten as

$$
\sigma_{11}=\frac{C_{11}}{1-C_{12} S_{21}-C_{13} S_{31}} \cdot \varepsilon_{11}
$$

where all $C_{i j}$ were computed previously, during the search for the wrinkle angle $\beta$. The compliance matrix components $S_{21}$ and $S_{31}$ are determined from the inverse of the stiffness matrix:

$$
\begin{aligned}
S_{21}= & \frac{C_{31} C_{23}-C_{21} C_{33}}{\mathbf{C}} \\
S_{31}= & \frac{C_{21} C_{32}-C_{31} C_{22}}{\mathbf{C}} \\
\text { with } \mathbf{C}= & C_{31} C_{12} C_{23}-C_{31} C_{13} C_{22}-C_{21} C_{12} C_{33} \\
& \quad+C_{21} C_{13} C_{32}+C_{11} C_{22} C_{33}-C_{11} C_{23} C_{32}
\end{aligned}
$$

While isotropic materials allow several possible solutions for $D_{e f f}$, in the case of anisotropic materials there is only one solution that satisfies Equation (5). In wrinkle-oriented coordinates the effective elasticity matrix is

$$
D_{\text {eff }}=\left[\begin{array}{ccc}
\frac{C_{11}}{\left(1-C_{12} S_{21}-C_{13} S_{31}\right)} & 0 & 0 \\
0 & 0 & 0 \\
0 & 0 & 0
\end{array}\right]
$$

where $D_{e f f, 11}$ is the rotated material stiffness, from Equation (6). Hence we can write

$$
D_{\text {eff }}=\left[\begin{array}{ccc}
E_{\text {rot }} & 0 & 0 \\
0 & 0 & 0 \\
0 & 0 & 0
\end{array}\right]
$$

$$
6 \text { of } 15
$$


Note that $D_{\text {eff }}$ is matrix of rank 1. In comparison, $D_{\text {eff }}$ found by Miller et al. for isotropic material (after rotation into wrinkling orientation) contains an additional component for the shear stiffness, and so has rank 2:

$$
D_{e f f}=\left[\begin{array}{ccc}
E & 0 & 0 \\
0 & 0 & 0 \\
0 & 0 & \frac{E}{4}
\end{array}\right]
$$

\section{Verification Test Cases}

\section{V.A. Experimental Validation of Viscoelastic Subroutine}

Young and coworkers ${ }^{22}$ conducted creep experiments on a flat-facet balloon structure with 48 gores and nominally $4 \mathrm{~m}$ diameter. The flat facet design uses gores whose widths correspond to the tendon spacing and as a consequence the structure contains no built-in lobing. The balloon was made of PBO and the same coextruded film that is used for ULDBs, with a thickness of 38 microns. The gores were joined via heat sealing. Similar in design to full-scale balloons, in this model the tendons were terminated with Brummel splices at both top and bottom end-fittings.

The structure was inflated five times to a very small differential pressure before the test series started. Additionally, two tests were carried out with the same structure at pressures of up to $100 \mathrm{~Pa}$ and $300 \mathrm{~Pa}$ before the experiment considered in this paper. After each test the balloon was left depressurized for at least 12 hours before the subsequent test, to allow viscoelastic recovery.

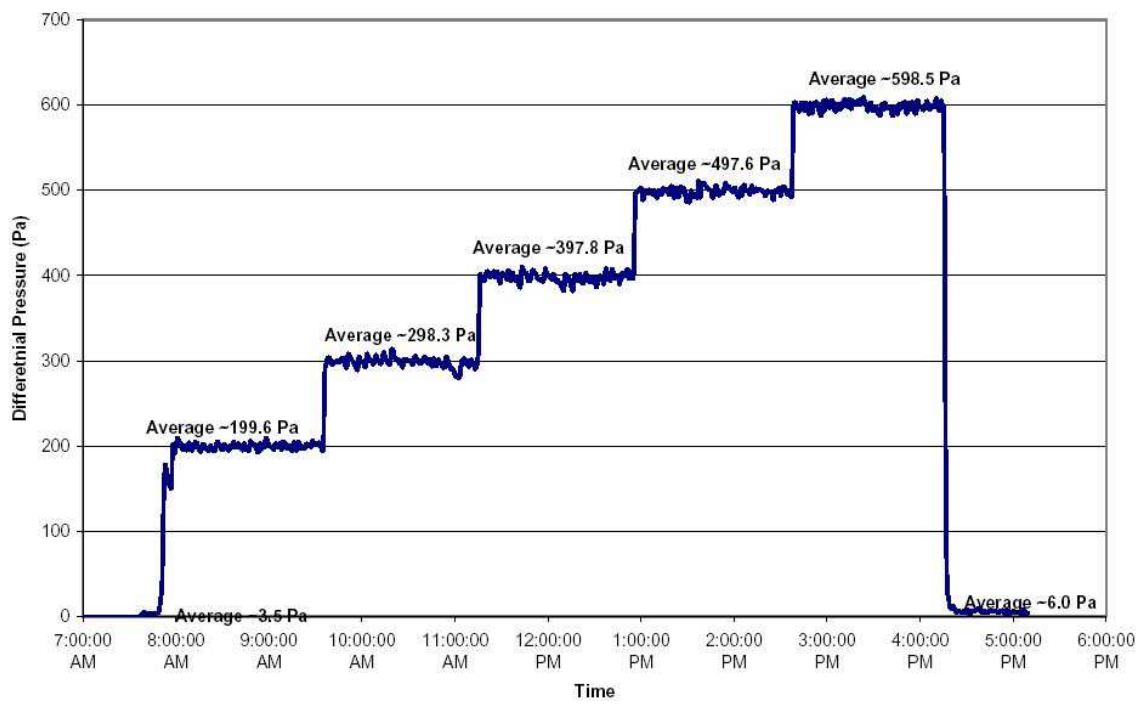

Figure 4. Differential pressure over time, from Cathey et al. ${ }^{2}$

Experimental creep measurements were made by means of photogrammetry using a setup of three cameras mounted on tripods (see figure 1). The balloon was hung in an open area and filled with a mixture of Helium and air. The structure was then pressurised in five increments and at each pressure level the pressure was maintained for approximately 90 minutes. Sets of three simultaneous photographs were taken of the targeted regions of the equatorial region of several lobes of the balloon, which had previously been fitted with photogrammetry targets, and the photos were used to determine the strains in the target regions, at the various differential pressures. The three-dimensional coordinates of the targets were determined with photogrammetry software, with a reported accuracy better than $0.063 \mathrm{~mm}$. The particular test that is considered in this paper took the structure to the desired maximum differential pressure level of $600 \mathrm{~Pa}$ in six increments. The applied pressure over time is plotted in Figure 4.

For the simulation a quarter of a gore was modelled in ABAQUS with triangular membrane elements (M3D3). The model consists of about 4400 elements, the mesh being finer along the edges of the gore and towards the end-fittings. Each tendon was allowed to move within a meridional plane of symmetry; the equator nodes were constrained to lie in the equatorial plane, while the apex points were allowed to move only along the axis of the balloon. For pressurisation the DLOAD command was used. The differential pressure 
levels were applied instantaneously in a step function, as opposed to the actual ramp functions used during the experiment.

\section{V.B. Anisotropic Wrinkling}

For verification of the anisotropic subroutine it was decided to use a pre-tensioned rectangular membrane. A uniform biaxial state of stress was set up by applying the stress $\sigma_{0}$ and the load $P=t h \sigma_{0}$ as shown in Figure 5. After pre-tensioning, in-plane bending moments $M$ were applied on the side edges, then $M$ was gradually increased. The stress $\sigma_{x}$ at the lower edge of the membrane eventually becomes compressive and a band of vertical wrinkles, of height $b$, forms. Details about the analytical solution are given in reference 18 . The membrane is partly wrinkled if

$$
\frac{1}{6} \leq \frac{M}{P h}<\frac{1}{2}
$$

where the ratio $b / h$ can be obtained from

$$
\frac{b}{h}=\frac{3 M}{P h}-\frac{1}{2}
$$

This test is run with both the new anisotropic wrinkling model and the proven isotropic wrinkling model by Miller et al. ${ }^{10}$ The anisotropic implementation is expected to produce the same results as the isotropic implementation (using the same isotropic material properties).

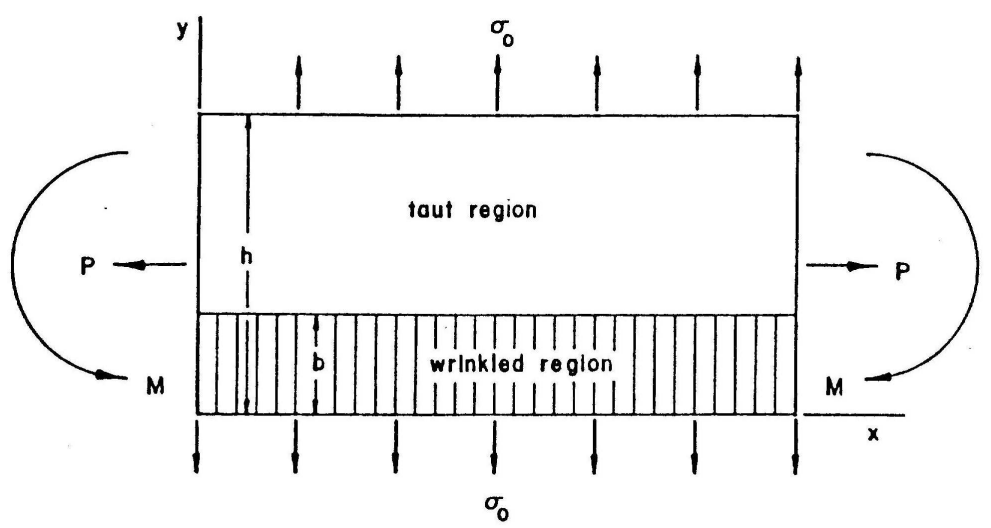

Figure 5. Flat membrane under biaxial stress and in-plane bending, from Miller et al. ${ }^{10}$

An $80 \mathrm{~mm} \times 160 \mathrm{~mm}$ rectangular membrane has been modelled with $16 \times 16$ triangular membrane elements (M3D3). A nominal stress $\sigma_{0}=2.0 \mathrm{MPa}$, a force $P=16 \mathrm{MPa}$, and a moment $M=300 \mathrm{Nmm}$ have been applied. From Equations (11) and (12), wrinkling is expected to begin for $M=213.3 \mathrm{Nmm}$; when the full moment is applied the wrinkled region has a width of $b=18.4 \mathrm{~mm}$.

The nodes along the left edge in Figure 5 were constrained in the $x$-direction and in addition the node at the center of the left edge was also constrained in the $y$-direction. All nodes along the edge on the right were kinematically tied in the $x$-direction to a dummy node, while the center of the edge were additionally tied in the $y$-direction to the dummy node. The dummy node was free to move in plane. This approach allows the force $P$ and the moment $M$ to be applied at a single node.

\section{V.C. Combination of Viscoelasticity and Wrinkling}

After the two subroutines, the viscoelastic model and the wrinkling model, had been verified separately as described in the previous two sections, an attempt was made to combine viscoelasticity and wrinkling into one implementation.

At the beginning of a time increment when the subroutine is called by ABAQUS a taut state of stress is assumed. Then the iterative algorithm of the viscoelastic subroutine is run to find the state of stress, for a taut element. Using the resulting stresses the element is checked for wrinkles (see section IV.A). If a wrinkled or slack state is obtained, the state of stress and the Jacobian matrix need to be modified. Alternatively if the element is found to be taut the viscoelastic subroutine continues as usual. In the case of a wrinkled element a slightly modified wrinkling algorithm is entered: after the wrinkling orientation is determined the 
Input variables:

From ABAQUS: time and strain increment Stored: stress history, old stresses, old reduce time

\section{Best guess for stress increments} based on current ABAQUS strain increments,

Use old nonlinearity parameters and reduced time
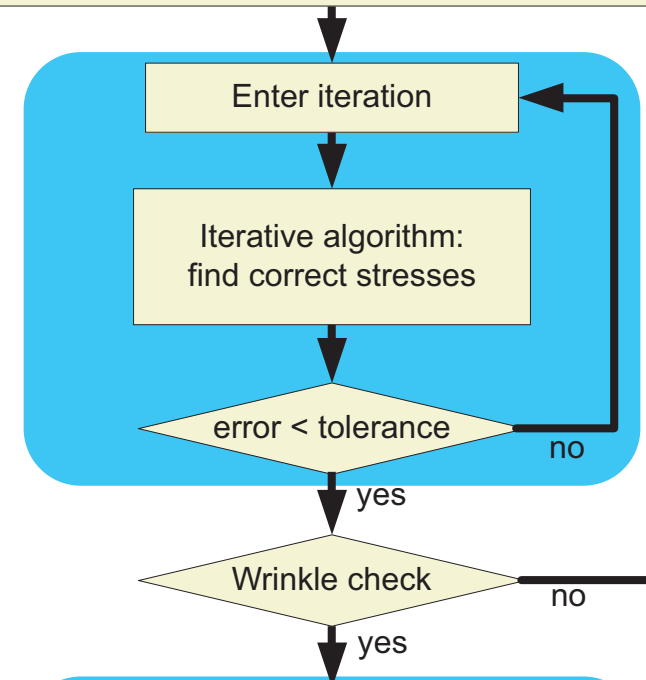

Enter uniaxial iteration

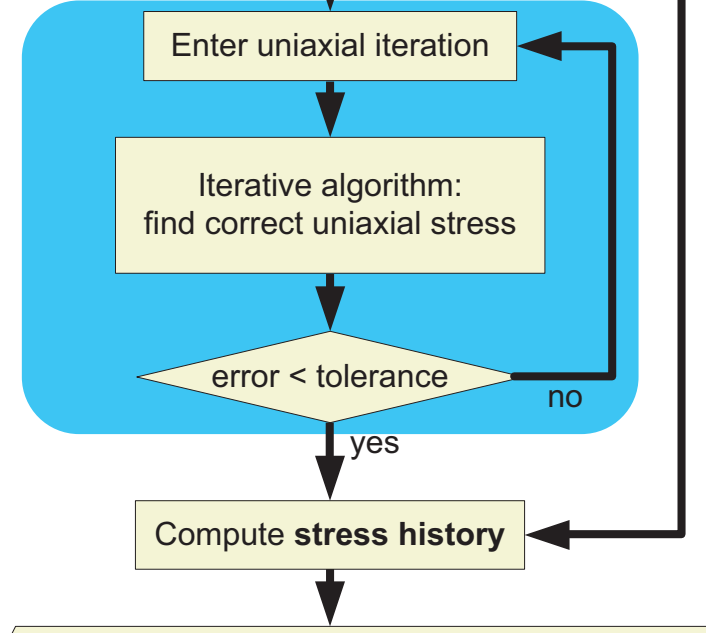

Output variables:

To ABAQUS: current stresses, Jacobian Store: stress history, stresses, reduced time

Figure 6. Combination of viscoelasticity and wrinkling. 
previously found stresses are discarded. Strains and history variables are rotated into the wrinkle orientation and the iterative algorithm that was previously used to find the stress state is run once again, but this time uniaxially. Once the correct uniaxial stress is found all of the variables are rotated back into the material orientation and the viscoelastic subroutine continues as usual. A schematic overview of the subroutine is depicted in Figure 7.

A cylindrical balloon structure was used to test this combined approach, since a cylinder can be assumed to have a uniform stress distribution and hence is a useful tool for testing the viscoelastic subroutine. ${ }^{3,4}$ Starting with a pressurised cylinder, the ends of the cylinder were brought closer together, in order to cause a series of hoop wrinkles to form; in the hoop direction a high uniaxial stress is maintained. Viscoelastic effects then cause the hoop strains to increase over time and, due to Poisson's ratio effects, the wrinkles disappear over time.

The cylinder had a diameter of $300 \mathrm{~mm}$ and height of $760 \mathrm{~mm}$. By symmetry, only half of a $90^{\circ}$ sector of the balloon was modelled, with triangular membrane elements (M3D3); a mesh of 60 elements in the hoop direction and 65 elements in the longitudinal direction was used. A uniform internal pressure of $1500 \mathrm{~Pa}$ was applied through the DLOAD command while the end-fittings were held in place. This resulted in a nominal hoop stress of $5.9 \mathrm{MPa}$. Next the end-fittings were brought closer together, each by $5 \mathrm{~mm}$, causing the axial stress to become compressive and wrinkles to form.

The pressurisation and the movement of the end-fitting was applied "instantaneously", in very short time increments $\left(\Delta t=1 \times 10^{-20} \mathrm{~s}\right)$, and was completed before the viscoelastic model took effect. The end movement was imposed in increments of $\Delta z=1 \mathrm{~mm}$ with a stabilisation factor of $1 \times 10^{-21}$, which was reduced to $1 \times 10^{-28}$ in a subsequent step.

\section{Results}

\section{VI.A. Flat Facet Balloon}

Young et al. ${ }^{22}$ have measured the creep strains on a flat facet balloon. This test was the first of this type and the results are plotted at the bottom of Figure 7. Measurements were taken from six different gores and for each gore the creep strains were averaged over the whole target area, covering about $250 \mathrm{~mm} \times 500 \mathrm{~mm}$. Overall, there is considerable spread in the results; it was concluded that variations in gore width were the source of this spread.

Based on the recorded pressure over time a simulation was run with a quarter of a gore using the viscoelastic subroutine presented in section III. Two sets of results are plotted at the top of Figure 7: the continuous line shows the creep strain predicted at the equator, at the center of the lobe; this is the maximum creep strain on the whole lobe. The dashed line is the average strain over a region somewhat smaller than that were the experimental results were averaged. Minor local irregularities were observed in the area along the edge of the gore and hence this region was not included in the average strain result. Including this area would have further decreased the average strains.

\section{VI.B. Flat Membrane}

To check that the anisotropic wrinkling approach is working, a pre-tensioned rectangular membrane under in-plane bending was modelled. For comparison to the results from the well-established isotropic implementation, both subroutines were used with the same loading and the same isotropic material properties. The results are compared in Figure 8. The left column shows the results from the isotropic implementation, while the right column shown the new anisotropic implementation. Figures 8 (a) and (b) depict the membrane right at the beginning of wrinkling, which occurred as expected at about $215 \mathrm{Nmm}$. Up to this moment the results from both subroutines are identical. Once wrinkling started the anisotropic subroutine requires high values of stabilisation (numerical damping) for convergence. In order to achieve convergence the remaining bending moment was applied in several increments. For each increment, after convergence had been reached, a subsequent step was run with lower numerical dumping while keeping the loading constant. Figure 8(c) shows the converged membrane with fully applied moment $M$ but still with considerable stabilisation. Figures $8(\mathrm{~d})$ and (e) depict the end results of both subroutines, where the stabilisation was sufficiently decreased to arrive at the same result as the isotropic subroutine. 

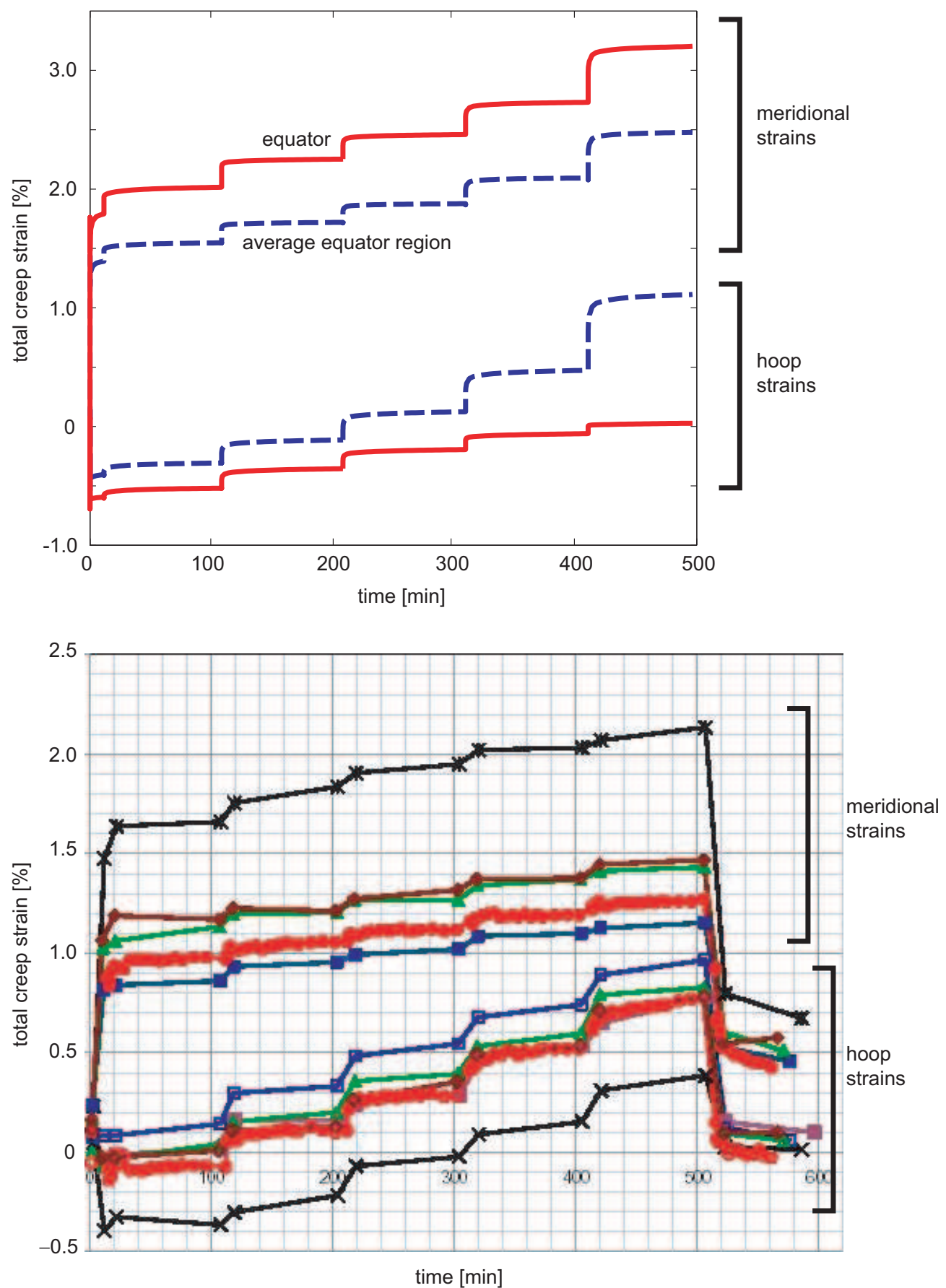

Figure 7. Predicted creep strains from viscoelastic subroutine and experimental results from. ${ }^{22}$ 
Isotropic Wrinkling Subroutine

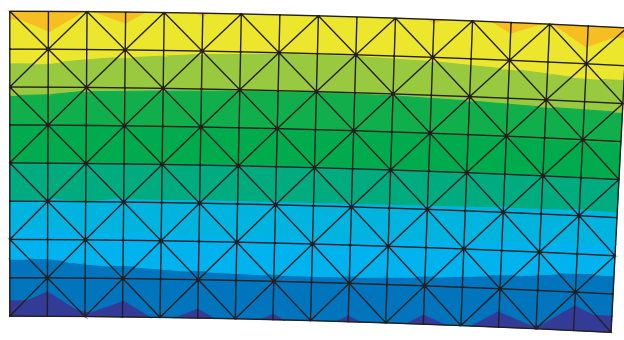

(a)
Anisotropic Wrinkling Subroutine

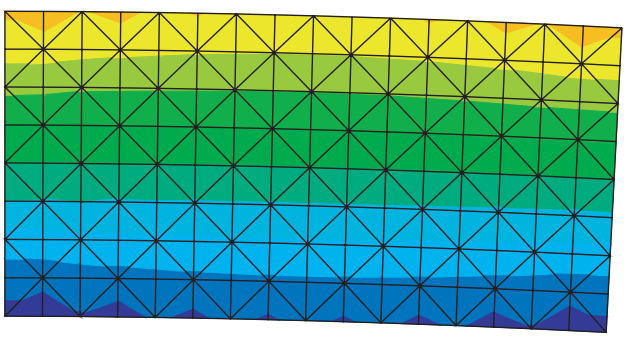

(b)

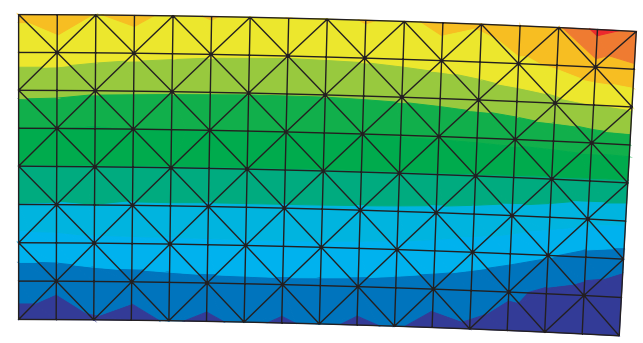

(c)

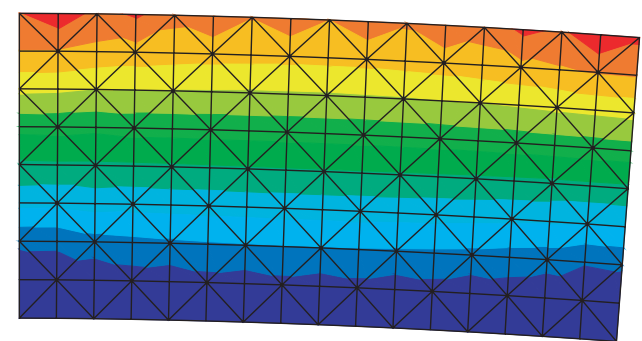

(e)

(d)

Figure 8. Comparison of isotropic and anisotropic wrinkling subroutines. 


\section{VI.C. Cylinder}

An attempt to combine the two separate implementations for viscoelasticity and wrinkling has been tested on a cylindrical structure. The ends of the cylinder were brought closer together in order to produce a uniform wrinkled state. Figures 9 and 10 show the results from this approach. The cylinder sections in Figure 9 show a quarter of a cylinder with the end shown on the left and the central section on the right. The contours describe the stresses in the axial direction. Figure 9(a) was taken after initial pressurisation and before the ends were moved. Hence there is still a biaxial state of stress. The changes seen in Figure 9(b) were seen when the end of the cylinder was moved with a high stabilise factor. These differences disappear in the next step as seen in Figure 9(c) after the stabilise factor was reduced again. Figure 9(d) shows the cylinder after 10 minutes creep. These images are related to stress states at particular times, shown in Figure 10 by means of the markers (a)-(d).

In general the cylinder behaved as expected: the wrinkles that were induced during the elastic phase $\left(t<1 \times 10^{-20} \mathrm{~s}\right)$ disappeared as soon as viscoelasticity took effect, due to Poisson's ratio's effects. However, numerical problems were observed during the analysis. This can be seen especially on the left end of the cylinder (in particular Figure 10(c)), where the stress in axial direction abruptly changes. Further investigation of this result is required.

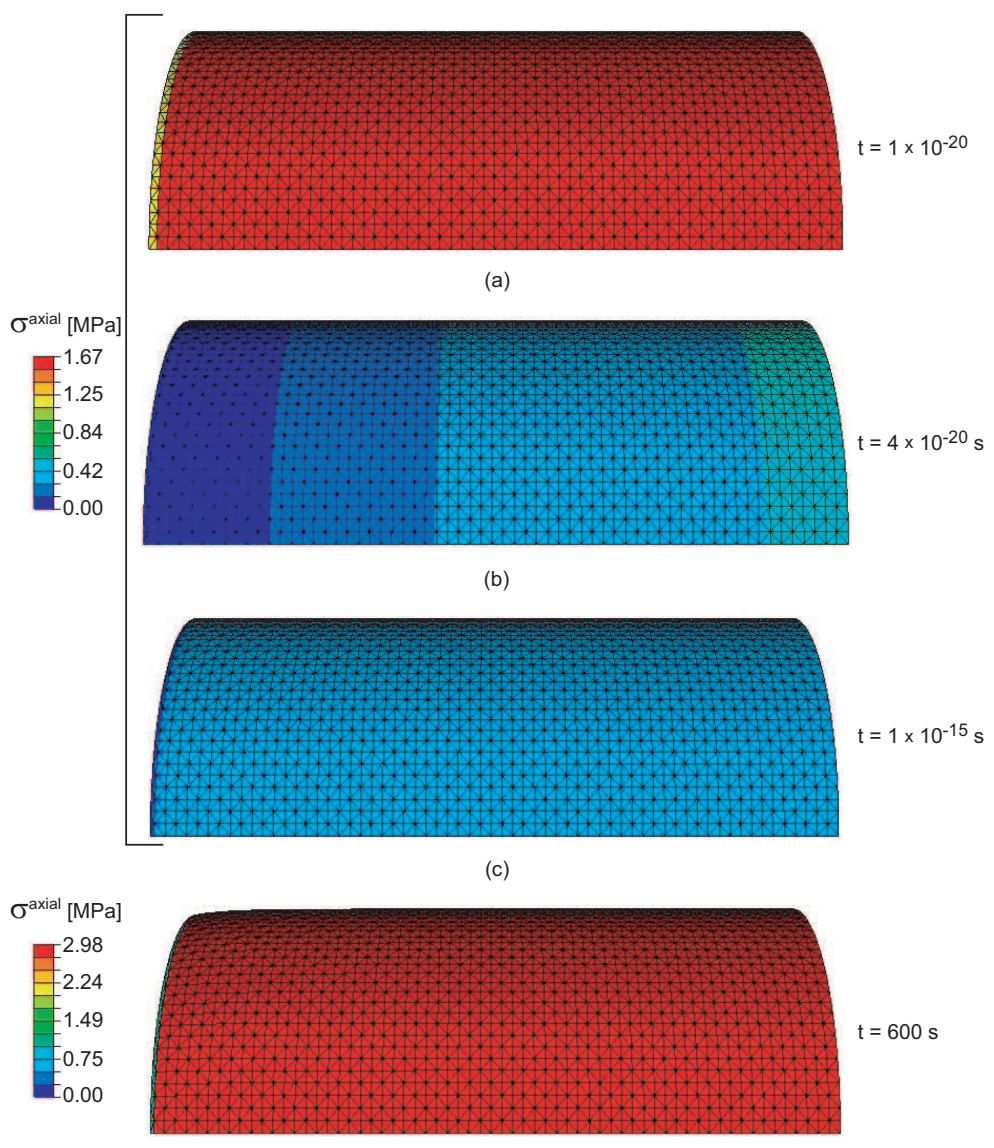

(d)

Figure 9. Axial stress over time in an initially wrinkled cylinder.

\section{Conclusion}

An iterative algorithm for the multiaxial formulation of Schapery's nonlinear viscoelastic material model has been presented. The algorithm has been implemented for displacement based finite element programs using the user-defined-subroutine interface available in ABAQUS. Using the viscoelastic material data from StratoFilm 372, published by Rand and co-workers, the numerical model has been applied to a flat facet 


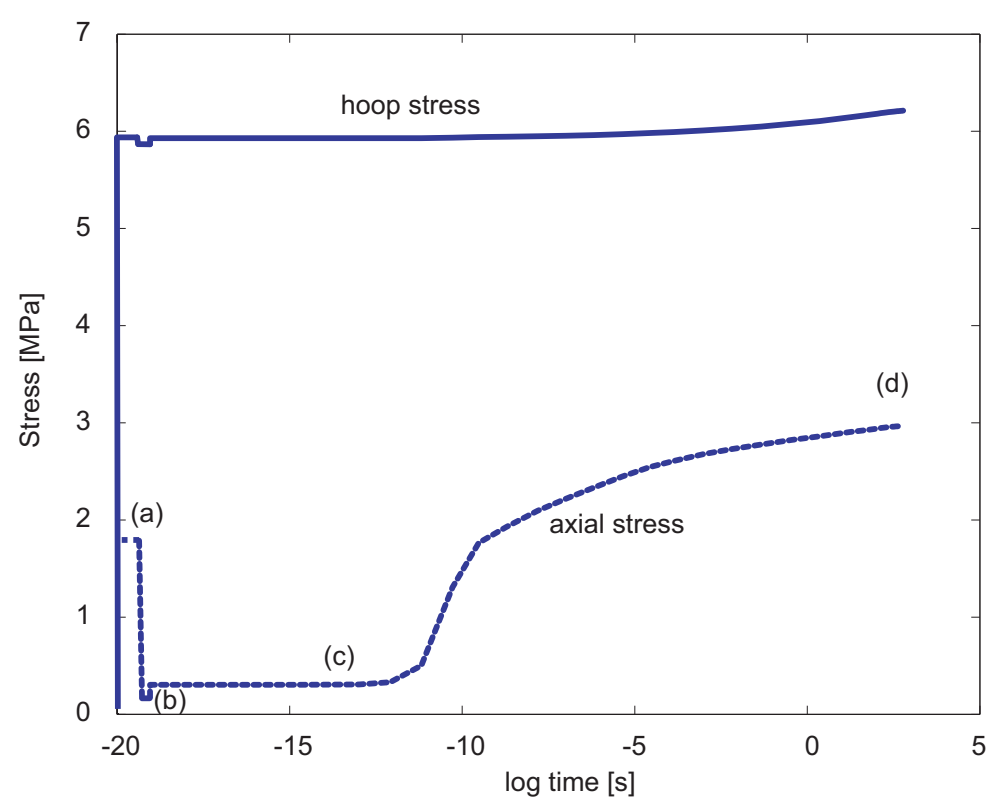

Figure 10. Stress variation over log time in an initially wrinkled cylinder.

balloon and predictions have been compared to experimental results. The predicted creep strains were higher than the experimental strains, mainly because experimental strains have been averaged over a larger area and so taking also into account areas with much lower strains. This trend has been shown by averaging the predictions over an area around the equator.

An attempt has been made at considering wrinkling in thin anisotropic films. An algorithm to find the wrinkle orientation and correct for a uniaxial state of stress has been presented and also implemented using the ABAQUS user-defined subroutine interface. While it has been found to produce correct results, convergence was achieved only after numerical damping had been introduced. Numerical damping was then reduced in subsequent steps.

The two separate implementations for viscoelasticity and wrinkling were combined into one subroutine. This approach was tested by means of a cylindrical balloon that formed uniform wrinkles in the hoop direction. The initially wrinkled cylinder behaved as expected: the wrinkles disappeared as soon as viscoelasticity took effect due to the Poisson's ratio effect. However numerical problems were observed and will need further investigation.

Finally, it should be noted that this paper is part of an ongoing effort. Some of the approaches presented will be refined in the future. In particular this includes the investigation of numerical instabilities and improvement of convergence when the wrinkling algorithm is used.

\section{Acknowledgments}

We thank Dr Jim Rand for continuing advice and for providing film material data to us, and Dr David Wakefield for his insightful suggestions. Financial support from the NASA Balloon Program Office (monitor: Mr Danny Ball) is gratefully acknowledged.

\section{References}

${ }^{1}$ Cathey, H.M. Test flights of the NASA ultra-long duration balloon. Advances in Space Research, 33, 1633-1641, 2004.

${ }^{2}$ Cathey, H.M., Sterling, J., Young, L.G., Garde, G.J., Pierce, D. UldB 48 Gore Flat Facet Balloon Strain Arrest Test Report. Report, Physical Science Laboratory and NASA Code 541, 2007.

${ }^{3}$ Gerngross, T., Xu, Y., And Pellegrino, S. Viscoelastic behaviour of pumpkin balloons. To appear in Advances in Space Research, 2007.

${ }^{4}$ Gerngross, T., And Pellegrino, S. Modelling of Anisotropic Viscoelastic Behaviour in Super-Pressure Balloons. 48th AIAA/ASME/ASCE/AHS/ASC Structures, Structural Dynamics and Materials Conference, AIAA-2007-1808.

${ }^{5}$ HaJ-Ali, R.M., AND Muliana, A.H. Numerical finite element formulation of the schapery nonlinear viscoelastic material 
model. International Journal for Numerical Methods in Engineering, 59, 25-45, 2004.

${ }^{6}$ Henderson, J.K., Caldron, G., And Rand, J.L. A nonlinear viscoelastic constitutive model for balloon films. AIAA 32nd Aerospace Sciences Meeting and Exhibit, AIAA-1994-638, 1994.

${ }^{7}$ KAng, S., AND Im, S. Finite element analysis of wrinkling membranes. Journal of Applied Mechanics, 64, 263-269, 1997.

${ }^{8}$ McCrum, N.G., Buckley, C.P., And Bucknall, C.B. Principles of Polymer Engineering, 2nd edition ed. Oxford Science Publications, Oxford, 2003.

${ }^{9}$ Miller, R.K., And Hedgepeth, J.M.. An algorithm for finite element analysis of partly wrinkled membranes. AIAA Journal 1982, 20, 12, 1761-1763, 1982.

${ }^{10}$ Miller, R.K., Hedgepeth, J.M., Weingarten, V.I., Das, P., Kahyai, S.. Finite element analysis of partly wrinkled membranes. Computers and Structures, 20, 1-3, 631-639, 1985.

${ }^{11}$ Pagitz, M., ANd Pellegrino, S. Computation of buckling pressure of pumpkin balloons. 47th AIAA/ASME/ASCE/AHS/ASC Structures, Structural Dynamics and Materials Conference, AIAA-2006-1803.

${ }^{12}$ Rand, J.L., Grant, D.A., And Strganac, T. The nonlinear biaxial characterization of balloon film. 34 th AIAA Aerospace Sciences Meeting and Exhibit, AIAA-96-0574, 1996.

${ }^{13}$ Rand, J.L., Henderson, J.K., And Grant, D.A. Nonlinear behaviour of linear low-density polyethylene. Polymer Engineering and Science, 36, 8, 1058-1064, 1996.

${ }^{14}$ Rand, J.L., And Sterling, W.J. A constitutive equation for stratospheric balloon materials. Advances in Space Research, 37, 11, 2087-2091, 2006.

${ }^{15}$ Roddeman, D.G., Drukker, J., Oomens, C.W.J., Janssen, J.D. The wrinkling of thin membranes: part 1 - theory. Journal of Applied Mechanics, 54, 884-887, 1987.

${ }^{16}$ Schapery, R.A. On the characterization of nonlinear viscoelastic materials. Polyner Engineering and Science, 9, 4, 1969.

${ }^{17}$ SCHAPERY, R.A. Nonlinear viscoelastic and viscoplastic constitutive equations based on themodynamics. Mechanics of Time-Dependent Materials, 1, 209-240, 1997.

${ }^{18}$ Stein, M., And Hedgepeth, J.M. Analysis of partly wrinkled membranes. NASA Technical Note D-813, 1961.

${ }^{19}$ Sterling, AND RAnd. Biaxial stress limit for ULDB film. AIAA 5th Aviation, Technology, Integration and Operations Conference (ATIO), AIAA-2005-7470, 2005.

${ }^{20}$ WAKefield, D. Numerical modeling of pumpkin balloon instability. AIAA 5th Aviation, Technology, Integration and Operations Conference, AIAA-2005-7445, 2005.

${ }^{21}$ Ward, I.M. Mechanical Properties of Solid Polymers, 2nd edition. John Wiley \& Sons, 1985.

${ }^{22}$ Young, L.G., Garde, G.J., Sterling, W.J. A practical approach for scientific balloon film strain measurement using photogrammetry. AIAA Balloon Systems Conference, AIAA-2007-2607, 2007. 